\title{
Reviewer Recognition 2017
}

Over the past year, just under 400 reviewers have contributed their time and expertise to ensure that the Journal of Spinal Cord Medicine brings you the best of the many submissions we receive. Without them, we would not be able to continue into the Journal's fifth decade of serving the community of spinal cord injury professionals. To all of those listed here, we say, "Thank you."

--The Editors

A. Gary Linassi

A. Martin

Aaron Phillips

Adonis Hijaz

Akmal Hafizah

Alan Diamond

Alberto Bazzocchi

Alberto Martinez Arizala

Alex Castro

Alex Ovechkin

Alicia Zukas

Allan Kozlowski

Allen Seftel

Altay Sencer

Amanda Botticello

Amit Amit

Amit Bhargava

Amitabh Jha

Ana Valeria Aguirre-Guemez

Andréane Richard-Denis

Andreas Demetriades

Andrew Guccione

Andrew Smith

Andrew Taylor

Angela Kuemmel

Angela Starkweather

Aninda Acharya

Anthony Burns

Anthony DiMarco

Anthony Lequerica

Antonio Montoto Marques

Arcangelo Barbonetti

Arthur Cytryn

Arthur Sherwood

Aruna Ganju

Arvind Ramanujam

Aslan Baradaran

Athanasios Kyriakides

Audrey Hicks
Barry McKay

Bethlyn Houlihan

Blair Calancie

Brett Gerstenhaber

Brittany Simone

Camila Almeida

Camila Battistuzzo

Camilo Castillo

Carla Nooijen

Carmelo Lucio Sturiale

Carmine Coscia

Carol Gibson-Gill

Carol Gibson-Gill

Casey Kandilakis

Catherine Wilson

Ceren Yarar

Charles Hubscher

Charles McCormack

Charlesnika Evans

Chelsea Pelletier

Chester Ho

Chris Gregory

Christina Papadimitriou

Christina Rapidi

Christine Fekete

Christopher Cardozo

Christopher Cirnigliaro

Christopher Harnish

Christopher Laohathai

Christopher West

Cindy Nowinski

Claire Kalpakjian

Clare Bassile

Colin Franz

Conti Alessio

Cristina Ehrmann

Daniel Credeur

Daniel de Souza

Daniel Hawiger 
Daniela Terson de Paleville

Danielle Rice

Dany Gagnon

David Ditor

David Dolbow

David Gater

David McMillan

David Monoky

Dawei Li

Delphine Labbé

Denis Raymond

Dennis Bourbeau

Dennis Cordato

Derry Dance

Dimitry Sayenko

Dobrivoje Stokic

Donald Dumford

Donald Stein

Dorothy Nary

Doug Ota

Douglas Lanska

Dzung Dinh

Eberval Figueiredo

Edelle Field-Fote

Edward Benzel

Edward Rohn

Egberto Moura

Elias Haddad

Elizabeth Ardolino

Elizabeth Broad

Elizabeth Dugan

Ellen Merete Hagen

Emad Ibrahim

Eric Garshick

Erkin Sonmez

Ervin Sejdic

EunKyoung Hong

Fatimah Ahmedy

Felicia Skelton

Frances Weaver

Frederique Courtois

Frederique Courtois

Gail Forrest

Gary Farkas

Gary Maerz

Gautam Mehta

George Alexiou

George Forrest

Gopalakrishnan Chittur Viswanathan

Graham Creasey

Gregory Holmes

Gregory Nemunaitis
Gregory Sawicki

Gregory Schilero

Griffin Baum

Guy Fried

Hannah Aura Shoval

Hany Morkos

Harvey Smith

Heather Flett

Hiroaki Matsumoto

Hirotaka Haro

Hongfei Xiang

Hongqi Zhang

Hongwu Wang

Hooman Azmi

Hossein Rouhani

Ian Rice

Inder Perkash

Ines Bersch-Porada

Inge Eriks-Hoogland

Jafar Kafaie

James Frisbie

James Harrop

James Milligan

James Walter

Jan Reinhardt

Jason Colizzo

Jay Menaker

Jayne Donovan

Jean Peduzzi

Jean-François Lemay

Jeanne Hoffman

Jeanne Zanca

Jeffery Johns

Jeffrey Berliner

Jeffrey Harrow

Jennifer Bunn

Jessica Bloomgarden

Jessica Lerch

Jesus Benito

Jie $\mathrm{Yu}$

Jill Wecht

Jitender Chaturvedi

Joëlle Flück

John Bach

John Cirillo

John Lavelle

John O'Neill

John Steeves

John Wheeler

Jonathan Strayer

José Ramírez Villaescusa

Jose Zariffa 
Joshua Yarrow

Joyce Fichtenbaum

Jude Delparte

Julia Patrick Engkasan

Julia Totosy de Zepetnek

Julian Cahill

Julie Gassaway

Julio Furlan

Jurgen Pannek

Justyna Swol

Karen Ethans

Karen Smith

Kari Keys

Kate Jones

Kath Bogie

Kathryn Tortorice

Kei Masani

Kendra Betz

Kent Campbell

Kevin Alschuler

Kevin Stroupe

Khalil Ghedira

Krista Best

Kristin Musselman

Kristine Cowley

Kyriakos Dalamagkas

Laila Mohsen

Lance Goetz

Laura O'Brien

Laura Rice

Laura Schopp

Laura Vogtle

Lawrence Vogel

Lee Stoner

Leili Dolatshahi

Leszek Herbowski

Lillian Stevens

Linda Ehrlich-Jones

Lisa Beck

Lisa Harvey

Lisa Ottomanelli

Lukas Grassner

Lynnette Jones

Maggi Budd

Mahboobe Mehrabani

Mahmoud AbdelRazek

Marc Babi

Marcel Dijkers

Margaret Finley

Maria Knikou

Marilyn Pacheco

Martin Forchheimer
Martin Reis

Mary Ann Richmond

Mary Slavin

Marylou Guihan

Massimo Venturelli

Matija Milosevic

Matthew Davis

Matthias Sczesny-Kaiser

Maureen Jennings

Meegan Van Straaten

Melissa Kaufman

Michael Fehlings

Michael Jones

Michael Keith

Michael Richardson

Michael Stillman

Michael Viega

Michael Wang

Milos Popovic

Ming Wu

Mirko Aach

Mohammad Hosein Ebrahimzadeh

Mohammad Alavinia

Mostafa Hosseini

Mufit Akyuz

Muhammad Abd-El-Barr

Murat Ekşi

Muthuraman Pandurangan

Naaz Kapadia

Nancy Chiaravalloti

Naser Asgari

Nataša Puzić

Nathan Hogaboom

Nathaniel Makowski

Nazi Derakhshanrad

Nicole Wagaman

NingNing Dou

Noam Harel

Nomathemba Taukobong

Nombeko Mshunqane

Nooredin Mohammadi

Oliver Cruciger

Olufemi Ajani

Pamela Fitzpatrick

Pamela Houghton

Parin Parmar

Patricia Heyn

Paul Arnold

Paula Geigle

Peter Felleiter

Peter Lim

Peter New 
Phil Weinstein

Philip Requejo

Philip Ullrich

Pierre Asselin

Pouran Faghri

Prithvi Shah

Purvi Sevak

Raja K.Kutty

Ralph Marino

Raman Malhotra

Ray de Leon

Rebecca Charbonneau

Ricardo Tanhoffer

Richard Bergman

Richard Bridges

Richard Krupkin

Riswan Hamid

Robert Heary

Robert James

Rodrigo Lopez-Gonzalez

Romel Mackelprang

Ronald Reeves

Rose Pignataro

Rüdiger Freudenstein

Ruth Marshall

Ryan McGrath

Ryan Solinsky

Ryan Sumrell

Sachin Watve

Sait Ozturk

Salah Rubayi

Salvador Cruz-Flores

Sandra Brotherton

Sangeeta Mahajan

Sanjay Kumar

Sara Lenherr

Sarah Munce

Seniz Akcay Yalbuzdag

Serge Marinkovic

Shane Sweet

Shaoping Hou

Shau Ping Lin

Shaun Gray

Shila Amarsheda

Shilpa Krishnan

Shinsuke Sakai
Shirley (Fitzgerald) Groer

Siamak Beheshti

Srikanth Tvm

Steven Brose

Steven Kirshblum

Sukhvinder Kalsi-Ryan

Sunil Sabharwal

Susan Garstang

Susan Jaglal

Susan Newman

Swati Mehta

T. Brett Reece

Talia Collier

Terrence Pugh

Tetsuya Abe

Themistoklis Tzatzairis

Thiago Heck

Thiago Heck

Thomas Bryce

Thomas Dixon

Thomas Kiser

Tim Bruns

Timothy Elliott

Timothy Hudson

Timothy Lavis

Todd Linsenmeyer

Tom Nightingale

Tomas Cervinka

Tracy Wall

Ümmühan Aktürk

Uriel Silva

Vanessa Bochkezanian

Vasileios Sakalis

Victoria Moreno-Manzano

Warren Grill

William Bauman

William Carter

Wise Young

Xan Nowakowski

Xiaojian Cao

Xifan Mei

xinmin Tang

Xueming Chen

Yi-Ting Tzen

Yuxi Chen

Zinal Chheda 